\title{
Evaluating crystal-orientation-dependent properties on polycrystalline tungsten: Example oxidation
}

\author{
Karsten Schlueter ${ }^{1,2}$, Martin Balden ${ }^{1}$, Tiago Fiorini da Silva ${ }^{1,3}$ \\ ${ }^{1}$ Max-Planck-Institut für Plasmaphysik, Boltzmannstr. 2, D-85748 Garching, Germany \\ ${ }^{2}$ Fakultät für Maschinenwesen, Technische Universität München, D-85748, Garching, Germany \\ ${ }^{3}$ Physics Institute of University of São Paulo - Rua do Matão, São Paulo, Brazil
}

\begin{abstract}
Various material properties, like oxidation, depend on the surface orientation of crystals. Most evaluations are focused on a few crystal orientations or using single crystals. A software tool written in python is introduced to correlate the thickness of the tungsten (W) oxide layer, i.e., the oxidation rate, and the individual single crystal grains with their surface orientation of polycrystalline $W$. The results are visualized in an inverse pole figure, which represent the oxidation rates for all crystal orientations. The use of polycrystalline $\mathrm{W}$ samples allow to analyze many different crystal orientations in a single experiment. Recrystallized, polished and polycrystalline W samples were precharacterized using electron backscatter diffraction in a scanning electron microscope (SEM). Subsequently, the samples were oxidized in a thermobalance. A confocal laser scanning microscope was used for measuring the height of the oxide layer, which is scaled to thickness of the oxide layer by using SEM images from focused ion beam prepared cross-sections. The previous data of oxidation of $\mathrm{W}$ in the temperature range of $720 \mathrm{~K}$ to $870 \mathrm{~K}$ were reanalyzed with the new analysis tool. At grain boundaries, the oxidation is influenced by the neighboring grains. To evaluate the magnitude of this effect on the oxidation, samples with different textures were oxidized and evaluated. The results for the different textures agree within the estimated error bars, demonstrating the effectiveness of the automated analysis method. W grains with $\langle 100\rangle$ surface orientation presented the highest oxidation rate.
\end{abstract}




\section{Introduction}

Properties like irradiation resistance [1], blistering [2], and oxidation [3] are influenced by the crystal lattice and consequently, its respective orientation of the surface. The sputter yield vary according to the crystal orientation easily by more than a factor of 10 [4]. In the past, some experiments were done with single crystals to evaluate properties, like sputtering, on the surface crystal orientation. Typically, in experiments that used polycrystalline samples only a few orientations were evaluated , e.g., for blistering [2]. Sometimes sputtering experiments were performed with different incident angles on a single crystal [4]. K. Nordlund et al. reported simulations on the effects of ion channeling for many different crystal orientations [5]. An experimental method capable of evaluate orientation dependent properties for many different crystal orientations is important for benchmarking such simulations. In the past, a practical method to assess the etching dependence on the crystal orientation was reported by $\mathrm{K}$. Sato et al. The authors used a single crystal silicon sphere and characterized its surface prior and subsequent to etching [6]. Because of the geometry of this single crystal sphere, the surface represents all crystal orientations and through a three-dimensional scan of the sphere, they could measure the etching behavior for all crystal orientations. Furthermore, it is unusual to have many single crystal samples compare to polycrystalline samples. In addition, preparing a sphere is not convenient and for some experiment, e.g., for sputtering, the sphere is not a useful geometry, because the incident angle of the ions is important [4]. In this study, polycrystalline, recrystallized tungsten (W) samples are used for developing a method to simultaneously measure properties for many different crystal surface orientations.

Studies on single crystals are time and effort consuming, expensive, and the extrapolation of the behavior to a textured sample can hardly be achieved. Conversely, the main advantage for using a flat polycrystalline samples is that many different crystal orientations are present on one sample and all orientations undergo the same conditions in the experiment, i.e., variations between different experimental runs are excluded.

The focus in this study is twofold: It lays (i) on obtaining an evaluation tool to correlate a property to many crystal orientations and (ii) on studying the crystal orientation dependence of the oxidation of W. Many research fields are interested in the use of $W$ oxides [7], while for some applications of $W$ at elevated temperature, oxidation needs to be avoided [8]. Especially in the field of semiconductors, e.g., for solar cells [9][10], photo catalysis [11], water splitting [12], sensors [13], and electrochromic devices [14] W oxides are of interest and useful. One example for an application with negative implications of $\mathrm{W}$ oxidation is the use of $\mathrm{W}$ in future nuclear fusion reactors. In case of accidental loss of coolant with simultaneous air ingress, $W$ can be oxidized [8, 15]. In such an accident [16], activated $\mathrm{W}$ oxide could be formed and sublimated leading to a potential safety issue if the volatile $\mathrm{W}$ oxide is released into the atmosphere. It has to be noted that the production of volatile $\mathrm{W}$ oxides could be strongly suppressed by the use of self-passivating $W$ alloys $[15,17,18]$.

Oxidation is a complex process because it depends on the absorption and diffusion of oxygen in the oxide layer and the bulk material, the diffusion of $\mathrm{W}$, and the formation of different phases of $\mathrm{W}$ oxide. Evidences for the influence of the crystal orientation on the oxidation process were already reported in a previous study [19].

Due to the enhancements in electron backscattering diffraction (EBSD) technology in the last years, which allows to measure up to several thousand crystal orientation per second, and the fast height measurement with the confocal laser scanning microscope (CLSM), a huge data base can be 
acquired. For the evaluation of millions of measured data points, a python tool has been written to combine the data from these two measurement systems. The python tool is able to merge the data sets and suitably visualize them in an inverse pole figure (IPF) (e.g., the oxidation rate correlated to the crystal orientation data). The data obtained in the previous study [19] is re-evaluated here using the new python tool. Additional oxidation experiments under the same condition were performed on different textured samples. The data sets were extended to the previous one, completing nonassessed regions in the IPF.

\section{Experimental}

For preparation and pre-characterization, first, hot rolled W samples of the purity of $99.97 \mathrm{wt} \%$ (Plansee SE, Austria) were recrystallized at $2000 \mathrm{~K}$ for 30 minutes under vacuum pressure below $1 * 10^{-7} \mathrm{mbar}$. Second, the samples were ground and electro-polished to achieve flat surfaces with height differences between the grains of less than $50 \mathrm{~nm}$ [19] [20]. Third, crystal orientation maps of polycrystalline $W$ samples were obtained by electron backscattering diffraction (EBSD) with an electron scanning microscope (FEl, Helios Nanolab 600). Thereafter, the samples were oxidized using a thermobalance (Netzsch, Jupiter, STA449 F1) at $870 \mathrm{~K}$ for $30 \mathrm{~min}$ forming a W oxide layer of a thickness of about $2-5 \mu \mathrm{m}$. Furthermore, the data for 720,820 and $870 \mathrm{~K}$ from the previous study were reanalyzed regarding all orientations. In addition, as a first test, an oxidation at $670 \mathrm{~K}$ for $30 \mathrm{~min}$ was performed to achieve a only several tens $\mathrm{nm}$ thin oxide layer.

The post-characterization included the measuring of the three dimensional surface profile of the samples using a confocal laser scanning microscope (CLSM) (Olympus, LEXT OSL4000), and the absolute thickness of oxide layer was determined for a limited number of grains by scanning electron microscopy (SEM) on focused ion beam (FIB) prepared cross-sections. Finally, using the thickness data from the FIB prepared cross-sections, the three dimensional surface profile data obtained by CLSM were converted to thickness of the oxide layer for the area pre-analyzed by EBSD. This procedure follows the previous study, and more details of the experiment and the calculation of the absolute thickness of the oxide layer can be found in [19].

\section{Merging of the data}

Merging image data obtained from different measuring systems is not only a displacement problem between two coordinate systems. 3D rotations, differences in magnification, and image distortions are just a few examples of problems that must be taken into account during the merging process. In [19], the data were merged by a manual procedure. In this work, we propose an automated procedure executed by a python tool developed to combine the data from two different microscopes. The algorithm of the procedure is visualized in the flow chart presented in fig. 1.

Fig. 2 illustrates a typical example of data merging (fig. 2(c)) of the height information (fig. 2(a)) and the crystal orientation (fig. 2(b)). The EBSD data are plotted by color-coding the three Euler angles, while the height information obtained by CLSM is visualized by using a gray scale and a shadow effect for a better grain visualization. In fig. 2 several markers prepared by FIB are visible, which are initially used as references to find the approximate alignment between the two images. White arrows point to those markers. The makers are $2 \mu \mathrm{m}$ wide and $20 \mu \mathrm{m}$ long, but are not the best references for the merging procedure. The intersections of three grain boundaries are far better and lead to better 
merging quality. Thus, the markers are used as a first approach to roughly merge the images, subsequently grain boundaries on both images are used in a second for optimize the merging process. After select reference points on both images, the tool calculates the new EBSD coordinates in the CLSM reference frame by using the python class scikit-image for the affine image transformation [21]. Thus, to each pixel in the EBSD map, a height is assigned, which is calculated by a bi-linear interpolation of the CLSM data. As the pixel size in the CLSM data is smaller than in the EBSD data, the data amount is reduced. An overlay of the two microscope pictures indicates the final result on the merging quality as shown in fig. 2(c).

If the final result on the merging quality is still not satisfactory, additional points may be used to calculate the reference frame transformation. The scikit-image class supports an unlimited number of distinctive points selected in both pictures to improve the affine transformation by correcting deformations. By experience, between 5 to 12 points are typically necessary for area measurement e.g. $1 \times 1 \mathrm{~mm}^{2}$.

After merging the new coordinates for the EBSD data, the three Euler angles, and the height information is combined and saved in one single output file. The merging with the python tool showed to be more accurate and much more efficient than the methodology used in the previous work [19]. This is because the manual merging only used the four FIB markers at the corners for the alignment of the images, and only a few points of selected orientation were analyzed. The new merging tool typically uses more than 4 points to perform a better alignment of the images, resulting in a better merging quality, and the whole image can be used to extract information on the influence of all crystal orientations on the oxidation process.

\section{Automated data selection and filtering}

The goal of the evaluation is to obtain the correlation between oxidation rate and surface orientation. A standard graph to visualize crystal orientation is an inverse pole figure (IPF). The IPF is a representation of all possible orientation in respect to one direction, here the surface normal " $\mathrm{z}$ " (IPFz), i.e., each combination of the 3 Euler angles is assigned to one point in the IPFz. Often thousands of measurements lay very close to each other. Therefore, the measurements are sorted in clusters. The orientation range in one cluster is around $0.2^{\circ}$. The uncertainty of the Euler angle determination and therefore the crystal orientation is assessed to a few degrees. This uncertainty does not originate only from the accuracy of the measuring system, but also from differences in the sample mounting into the measuring system. Several areas on different samples are measured and these data are combined to one data set, i.e., IPFz plot. All IPFz plots contain millions of measured data points. Therefore, it is reasonable to apply filters to enhance the data quality.

The first quality enhancement is performed on the EBSD data. A typical error is the miss-indexing by the automated pattern solving algorithms leading to wrong Euler angles. This leads mostly to isolated indexed data point in the orientation map (spikes), but for some specially orientated grains, the patterns for two orientation are very similar and the solution tends to oscillate between both. In fig. 2(b), a few of these grains having different solutions are marked by a black circle. The oscillation is visualized by two different colors (patchwork grains). Therefore, a noise filter was applied on the EBSD data, which bins $2 \times 2$ pixels and calculates the variance of the position in the IPFz plot. If the variance is bigger than 30 pixel, which correspond to a miss orientation angle of around $6^{\circ}$, all 4 points will be removed from the data set. The binning and removing of the data points is once 
repeated, but the starting position of the binning process is moved by one pixel to the right and one pixel down. So, if one EBSD data point is a spike, 6 data points next it will be removed. In addition to the spikes and the patchwork grains, the data points at the grain boundaries, which are not reliable, will be partially erased, too. Thereby, this noise filter will remove correct and wrongly indexed data points.

For data sets evaluated in this work, typically one pixel in the IPFz plot results from several thousand of measurements as visualized in fig. $3(\mathrm{~d}-\mathrm{f})$. Therefore, the standard derivation of the height values for all these measurements was chosen as an indicator for the statistical amount of variation between the measurements. The standard derivation is plotted without and with the above described noise filter in fig. 4. The reduction due to the noise filter is $20 \%(100 \mathrm{~nm})$, which is caused by the wrong indexed EBSD patterns and the partially removed data points along the grain boundaries.

The oxide layer variation at the grain boundaries is shown in fig. 2(c). The grain boundaries are only partially removed by the noise filter of the EBSD data, i.e., the influence of the boundary extents more than only some pixel in the orientation map into the grains. Therefore, the median instead of the mean of the height values was taken for each cluster (median filter), which is used for IPFz plot as shown in fig. 3.

Since the cluster size of around $0.2^{\circ}$ is an oversampling of the data, the neighboring pixels in the IPFz plot should be similar. Consequently, again the median of thickness value from each pixel in the IPFz plot with its direct eight neighboring pixels was taken as a smoothing filter. This erases spikes in the IPFz plot.

Some crystal orientation are simply not found on the analyzed area of the polycrystalline $\mathrm{W}$ samples used in this study, which result in white areas in fig. 3. Isolated pixels exist often in the white areas which result from less than 10 measurements. These pixels exhibit an unusual oxidation rate and are assessed as unreliable. As over 10 million measurements are evaluated for fig. 3 and a grain results in at least several 100 measurements of the crystal orientation and of the heights, an additional filter is applied to remove these pixels. Each pixel in the IPFz plot resulting from less than 10 measurements will be excluded in the IPFz plot (cut-off filter). Fig. 3(c) shows the final result after applying the noise filter to the EBSD data, taking the median of all measurement assigned to each pixel in the IPFz plot (median filter) and applying the smoothing filter and the cut-off filter (see fig. 1). 


\section{Oxidation in respect to the surface orientation}

As a cross check of the automated methodology developed here, the data of the previous studies [19] were re-analyzed with the new analyzing tool. The thickness variation of the oxide layer was investigated for different temperatures and for the same mean thickness of the oxide layer, which means that the oxidation time was adjusted. For all 3 temperatures, $720 \mathrm{~K}, 820 \mathrm{~K}$, and $870 \mathrm{~K}$, the grain dependent oxidation showed similar behavior for over thousand evaluated grains on each sample, i.e., the oxidation rates vary with surface orientation in the same fashion for all 3 temperatures. This means that the IPFz plots for $720 \mathrm{~K}$ and $820 \mathrm{~K}$ are - in the frame of the lower statistic of crystal orientation and height data - very similar to one for $870 \mathrm{~K}$ (fig. 3(c)).

In order to clarify if the orientation of neighboring grains influences the oxidation (fig. 2(c)), e.g., via lateral increased oxygen diffusion, $\mathrm{W}$ samples with different texture are of interest. Therefore, two W surfaces were selected, which exhibit different texture [22], i.e., some crystal orientations dominate the surface. One surface has more grains with surfaces close to $<100>$ orientation, which is called texture $A$, and another surface has more grains with surfaces close to $\langle 110\rangle$ and $\langle 100\rangle$ orientation, which is called texture B. In fact, the two used samples are from the same process batch and each sample exhibits both textures, one on the front and one on the back side [22]. By repeating the oxidation experiment with these two textures with a new, faster CMOS-based EBSD detector (Oxford Instr., Symmetry), a higher diversity of grain orientations is measured. Fig. 3(d) and (e) give the number of measuring points evaluated on each surface versus the surface orientation and, therefore, visualize the texture $A$ and texture $B$. Both samples with texture $A$ and texture $B$ show similar oxidation dependencies, which is visible in the separated plots in fig. 3(a) and (b). A second cross check was performed on the standard deviation. The standard deviation was visualized separately for texture $A$ and $B$ (not shown) and compared with the standard deviation for all measurements (fig. 4(b)). All three plots of the standard deviation are similar.

This agreement indicates that the automated methodology is robust and that the data of both samples can be combined to produce a more complete IPFz plot, as presented in fig. 3(c). This figure includes above $2.5 \times 10^{7}$ measuring points and shows the oxidation dependency for approximately all crystal orientations. The amount of measurements, this is the sum of texture $A$ and $B$, is shown in fig. 3(f). The oxide layer in fig. 3(c) is up to $5 \mu \mathrm{m}$ thick and the temporal weight increase in the thermobalance behavior is parabolic which indicates an oxygen diffusion driven oxidation. A crystalline structure could be an explanation for different oxygen diffusion rates though the $\mathrm{W}$ oxide layer. Therefore, specific investigations are performed for resolving the oxide phase in the sub- $\mu \mathrm{m}$ lateral length scale. Unfortunately, the available XRD has a far bigger beam spot and therefore it is not possible to distinguish between different oxide phases at different grains.

An ion picture is normally dominated by the crystal lattice contrast. In order to study whether the oxides depend on the crystal orientation, $\mathrm{a} \mathrm{Ga}^{+}$-ion pictures by detecting secondary electrons of an oxidized area are taken and an example is shown in fig. $5(b)$. In this picture, different brightnesses between the single oxidized grains are clearly visible. Additionally, EBSD measurements were tried, but the Kikuchi patterns were deformed and their evaluation was not possible, i.e., none of the theoretical patterns of the possible crystallographically oxide phases fits. Nevertheless, since Kikuchi patterns were visible, this is another indication for a crystalline structure in the oxide layer, which can influence the diffusion of oxygen through the oxide layer. A second interesting point is that scratches residueing from the grinding (fig. $5(\mathrm{a})$ ) are still visible on a up to $5 \mu \mathrm{m}$ thick oxide layer in fig. 5 (b). 
This shows that the oxide layer grows up from the $\mathrm{W}$ surface and the $\mathrm{W}$ oxides are influenced by the W surface. This means that roughness through the grinding or deformation of the surface influence the oxidation continuously and it is not a temporal phenomenon at the starting phase of the oxidation.

In this study, the first aim was the development of the method for measuring properties for thousands different crystal orientations in one experiment. The developed method shows for different textures similar distribution of the oxidation rate to the crystal orientation. For a several $\mu \mathrm{m}$ thick oxide layer, the grains with the $\langle 100\rangle$ surface orientation have the highest oxidation rate of all orientations, which is two times higher than those with the lowest oxidation rate of all orientations. The grains with $<111>$ surface orientation belong to those with lowest oxidation. This orientation dependence is also found at $720-870 \mathrm{~K}$ for oxide layers of 2-5 $\mu \mathrm{m}$ thickness. The introduced method can be used to investigate sputtering, etching or other properties in dependence to the crystal orientation.

\section{Discussion of oxidation}

Oxidation is a process which has several sub-processes like diffusion or absorption. At the starting point the W surface was electro-polished, but has still a few small scratches. These scratches were visible on a several $\mu \mathrm{m}$ thick oxide layer, as shown in fig. 5. As reported in [23], oxygen diffused through the oxide layer and $\mathrm{W}$ oxides are formed at tungsten surface. The surface information of the $\mathrm{W}$, like scratches are transferred to the oxide layer which could influence the oxidation behavior. Also, it is possibly that the crystal lattice information of the $\mathrm{W}$ is transferred to the $\mathrm{W}$ oxides which would have an influence on the diffusion of oxygen.

There are several hints that the grain dependent oxidation in the current studies is influenced by the diffusion of oxygen through crystalline $\mathrm{W}$ oxide. First, the temporal weight increase of the oxidation indicates a parabolic behavior. Second, Kikuchi patterns on the W oxide were observed with the EBSD system, but they were ambiguous. An evaluation of these patterns are not possible, i.e., no crystallographical oxide phase could be assigned. At least they showed a crystalline structure, which could influence the diffusion. Third, a Ga ion induced SE picture is dominated by a crystal orientation contrast (fig. 5(b)). The oxide grains with the lower brightness belong to $W$ grains with the $\langle 100\rangle$ orientation. An interpretation of these contrast data is speculative, because the $\mathrm{W}$ oxide crystallographical phase could be different at room temperature compare to the oxidation target temperature. The most stable $\mathrm{W}$ oxide is $\mathrm{WO}_{3}$, and has an orthorhombic crystal lattice at $870 \mathrm{~K}$, but at room temperature it could reduced to, e.g., a monoclinic crystal lattice [24]. Since the Ga-ion induced secondary electron picture (fig. $5($ b) ) is dominated by the crystal lattice contrast, it indicates W oxides with different crystal orientations.

During the evaluation the median is taken and filters are applied, like the noise filter, to reduce the effects of the neighboring grains. For cross checking the influence of the diffusion by neighboring grains and on the applied filters, the oxidation experiment is repeated with a differently textured $\mathrm{W}$ sample. The idea is that on average the neighboring grains have a differnet orientation and influence each other. Since the boundary area of the grain is in the $\mu \mathrm{m}$ range, a cross check is important, e.g., a circular grain with a diameter of $10 \mu \mathrm{m}$ can be separated in an inner cycle and a ring. The inner cycle with the radius of $3.5 \mu \mathrm{m}$ has a similar area as a ring with a boundary area between a radius $3.5 \mu \mathrm{m}$ to $5 \mu \mathrm{m}$ (boundary distance $1.5 \mu \mathrm{m}$ ). This means that the developed method could deliver a texture 
dependent result, because grains around $10 \mu \mathrm{m}$ would delivers wrong heights if most of the area (around $1.5 \mu \mathrm{m}$ from the grain boundary) is influence by grain boundaries. One sample with many W crystal grains with the $\langle 110\rangle$ orientation and therefore, another sample with many W crystal grains with $\{100\}$ orientation were oxidized. Both textured samples reveal similar crystal dependent oxidation behavior as shown in fig. 2(b) and (c). This could be because the diffusion up to $5 \mu \mathrm{m}$ perpendicular to the surface is the dominant diffusion direction and does not laterally diffuse as much as perpendicular to the surface. An explanation could be that the gradient of the amount of $\mathrm{O}_{2}$ and $\mathrm{O}$ in the oxide layer is perpendicular to the $\mathrm{W}$ bulk surface, because at the bulk surface $\mathrm{W}$ oxide is formed, resulting in the preferred diffusion direction. The grain boundaries influence the oxidation sometimes in the $\mu \mathrm{m}$ range as shown in fig. 2(c) (also [19] fig. 3), but the grains are big enough and therefore the result is not influenced by grain boundaries and the texture of the sample after applying all filters.

The diffusion of oxygen through the oxide layer to the $\mathrm{W}$ surface starts after a short time of the oxidation (less than a minute). For avoiding that the oxidation process is dominated by oxygen diffusion, a thin oxide layer is required. For a nm thin oxide layer, oxygen interacts with strongly reduced diffusion time with the $\mathrm{W}$ surface and the diffusion is not the limited process. For achieving a controlled $\mathrm{nm}$ thin oxide layer, the oxidation temperature is reduced to $670 \mathrm{~K}$. First measurements on FIB prepared cross-sections show that one of thickest oxide layers of around $50 \mathrm{~nm}$ occurs on the $W$ grains with the orientation $<111>$. This observation fits to the arguments of Ligenza [25]. He oxidized silicon with different crystal orientations and argues with the number of bonds is important between the Si-Si atoms. The $<111>$ W orientation has the lowest atomic surface density for the low indexed surfaces. The fastest oxidation rate of the $<111>$ orientation for a several $\mathrm{nm}$ thick oxide layer is in contrast to the oxidation rate for a several $\mu \mathrm{m}$ thick oxide layer, for which the <111> exhibits to the lowest oxidation rate. In other words, forming a several hundreds of $\mathrm{nm}$ thick oxide layer, the oxidation rate of the $<111>$ orientation reduces stronger than the other orientations, and the $<111>$ orientation has then the lowest oxidation rate compared to other orientations. The reason could be the diffusion of oxygen through the oxide layer. For resolving a $\mathrm{nm}$ thin oxide layer over thousands of crystal orientations another thickness measuring technique must be apply, which is planned for the future.

\section{Summary}

The main goal of this work was to develop a tool for correlating the data of two measuring system using different physical principles. Millions of data points are combined, which makes it reasonable to apply filters to enhance the quality, even if many high quality data points are lost together with the lower number of unreliable ones.

The developed python tool combines and evaluates the thickness of the oxide layers for all crystal orientations and provides the correlated data in an inverse pole figure. For a few $\mu \mathrm{m}$ thick oxide layer, the absolute thickness of the oxide layer, i.e., the oxidation rate, varies by a factor of two between the $<100>$ and $<111>$ orientation of the $W$ sample in a temperature range between $720 \mathrm{~K}$ to $870 \mathrm{~K}$. The oxidation rate of the low indexed surfaces as well as for the $<210>$ and $<211>$ crystal orientation is similar to the study in [19]. The distribution of the oxidation rate versus the crystal orientation is very similar on different textured samples, which means that the influences of the neighboring grains is negligible and, therefore, the lateral oxygen diffusion.

The secondary electron picture resulting from a Ga+ ion beam shows different contrast in the $\mathrm{W}$ oxides, which could hint to the crystal structure of the W oxide. Also, the EBSD measurement on the oxide layer shows Kikushi patterns, but the crystal orientation of the W oxide could not determined, 
because the Kikushi patterns were ambiguous. At least their observation is an indication that the tungsten oxide has partially a crystal structure.

After the successful demonstration of the usefulness of the method of combining different data sets, the introduced method will be applied to investigate different kinds of topics, as crystal orientated sputtering or crystal orientated etching. Furthermore, the studies of oxidation will be continued. It is planned to study the temporal behavior of oxidation, because first measurements on samples with $50 \mathrm{~nm}$ thin oxide layers ( 30 minute at $670 \mathrm{~K}$ ) on FIB prepared cross-sections show a different crystal dependent oxidation behavior. 


\section{Acknowledgement:}

This work has been carried out within the framework of the EUROfusion Consortium and has received funding from the Euratom research and training program 2014-2018 and 2019-2020 under grant agreement No 633053. The views and options expressed herein do not necessarily reflect those of the European Commision.

\section{References}

[1] Garrison, L. M., and Kulcinski, G. L. Irradiation resistance of grains near $\left\{\begin{array}{ll}0 & 0\end{array}\right\}$ on polycrystalline tungsten under $30 \mathrm{keV} \mathrm{He+} \mathrm{bombardment} \mathrm{at} 1173$ K. Physica Scripta, 2014; (T159), 014020.

[2] Jia, Y. Z., De Temmerman, G., Luo, G. N., Xu, H. Y., Li, C., Fu, B. Q., and Liu, W. Surface morphology and deuterium retention in tungsten exposed to high flux D plasma at high temperatures. Journal of Nuclear Materials, 2015; 457, 213-219.

[3] Alimov, V. K., Tyburska, B., Balden, M., Lindig, S., Roth, J., Isobe, K., and Yamanishi, T. Surface morphology and deuterium retention in tungsten oxide layers exposed to low-energy, high flux $D$ plasma. Journal of Nuclear Materials, 2011; 409(1), 27-32.

[4] Roosendaal, H. E. Sputtering yields of single crystalline targets. In Sputtering by particle bombardment I (pp. 219-256). Springer, Berlin, Heidelberg, 1981;

[5] Nordlund, K., Djurabekova, F., and Hobler, G. Large fraction of crystal directions leads to ion channeling. Physical Review B, 2016; 94.21, 214109.

[6] Sato, K., Shikida, M., Matsushima, Y., Yamashiro, T., Asaumi, K., Iriye, Y., and Yamamoto, M. Characterization of orientation-dependent etching properties of single-crystal silicon: effects of $\mathrm{KOH}$ concentration. Sensors and Actuators A: Physical 64.1 1998; 87-93.

[7] Bignozzi, C. A., Caramori, S., Cristino, V., Argazzi, R., Meda, L., and Tacca, A. Nanostructured photoelectrodes based on $\mathrm{WO}_{3}$ : applications to photooxidation of aqueous electrolytes. Chemical Society Reviews, 2013; 42(6), 2228-2246.

[8] Smolik, G. R., Piet, S. J., and Neilson Jr, R. M. Predictions of Radioactive Tungsten Release for Hypothetical ITER Accidents. Fusion Technology, 1991; 19(3P2B), 1398-1402.

[9] Varghese, O. K., Paulose, M., and Grimes, C. A. Long vertically aligned titania nanotubes on transparent conducting oxide for highly efficient solar cells. Nature nanotechnology, 2009; 4(9), 592597.

[10] Hara, K., Zhao, Z. G., Cui, Y., Miyauchi, M., Miyashita, M., and Mori, S. Nanocrystalline electrodes based on nanoporous-walled $\mathrm{WO}_{3}$ nanotubes for organic-dye-sensitized solar cells. Langmuir, 2011; $27(20), 12730-12736$.

[11] Yu, J., and Qi, L. Template-free fabrication of hierarchically flower-like tungsten trioxide assemblies with enhanced visible-light-driven photocatalytic activity. Journal of hazardous materials, 2009; 169(1), 221-227.

[12] Lai, C. W., and Sreekantan, S. Incorporation of $\mathrm{WO}_{3}$ species into $\mathrm{TiO}_{2}$ nanotubes via wet impregnation and their water-splitting performance. Electrochimica acta, 2013; 87, 294-302. 
[13] Wang, X., Miura, N., and Yamazoe, N. Study of $\mathrm{WO}_{3}$-based sensing materials for $\mathrm{NH}_{3}$ and $\mathrm{NO}$ detection. Sensors and Actuators B: Chemical, 2000; 66(1), 74-76.

[14] Berger, S., Tsuchiya, H., Ghicov, A., and Schmuki, P. High photocurrent conversion efficiency in self-organized porous $\mathrm{WO}_{3}$. Applied Physics Letters, 2006; 88(20), 203119.

[15] Koch, F., and Bolt, H. Self passivating W-based alloys as plasma facing material for nuclear fusion. Physica Scripta, 2007; (T128), 100.

[16] Maisonnier, D., Cook, I., Pierre, S., et al., N. The European power plant conceptual study. Fusion Engineering and Design, 2005; 75, 1173-1179.

[17] Calvo, A., García-Rosales, C., Ordás, N., Iturriza, I., Schlueter, K., Koch, F., Pintsuk, G., Tejado, E. and Pastor, J. Y. Self-passivating W-Cr-Y alloys: characterization and testing. Fusion Engineering and Design. 2017; 124, 1118-1121.

[18] Klein, F., Wegener, T., Litnovsky, A., Rasinski, M., Tan, X. Y., Gonzalez-Julian, J., Schmitz, J., Bram, M., Coenen, J.W. and Linsmeier, C. Oxidation resistance of bulk plasma-facing tungsten alloys. Nuclear Materials and Energy.2018; 15, 226-231.

[19] Schlueter, K., Balden, M. Dependence of oxidation on the surface orientation of tungsten grains. International Journal of Refractory Metals and Hard Materials 79 2019; 102-107.

[20] Manhard, A., Matern, G., and Balden, M. A step-by-step analysis of the polishing process for tungsten specimens. Practical Metallography, 2013; 50(1), 5-16.

[21] Python library Download-link: https://scikit-image.org/. 2018; Version: 0.14 .1

[22] Manhard, A. Balden, B., and Elgeti, S. Quantitative microstructure and defect density analysis of polycrystalline tungsten reference samples after different heat treatments. Practical Metallography Praktische Metallographie, 2015; 52:437- 466.

[23] Klein, F., Wegener, T., Litnovsky, A., Rasinski, M., Tan, X., Schmitz, J., Linsmeier, C., Coenen, J.W., Du, H., Mayer, J. and Breuer, U. On Oxidation Resistance Mechanisms at $1273 \mathrm{~K}$ of Tungsten-Based Alloys Containing Chromium and Yttria. 2018; Metals, 8(7), 488

[24] Lassner, E. and Schubert, W. D. Tungsten: Properties, Chemistry, Technology of the Elements, Alloys, and Chemical Compounds. Springer US, 1999. ISBN 9780306450532.

[25] Ligenza, J. R. Effect of crystal orientation on oxidation rates of silicon in high pressure steam. The Journal of Physical Chemistry, 1961; 65(11), 2011-2014. 


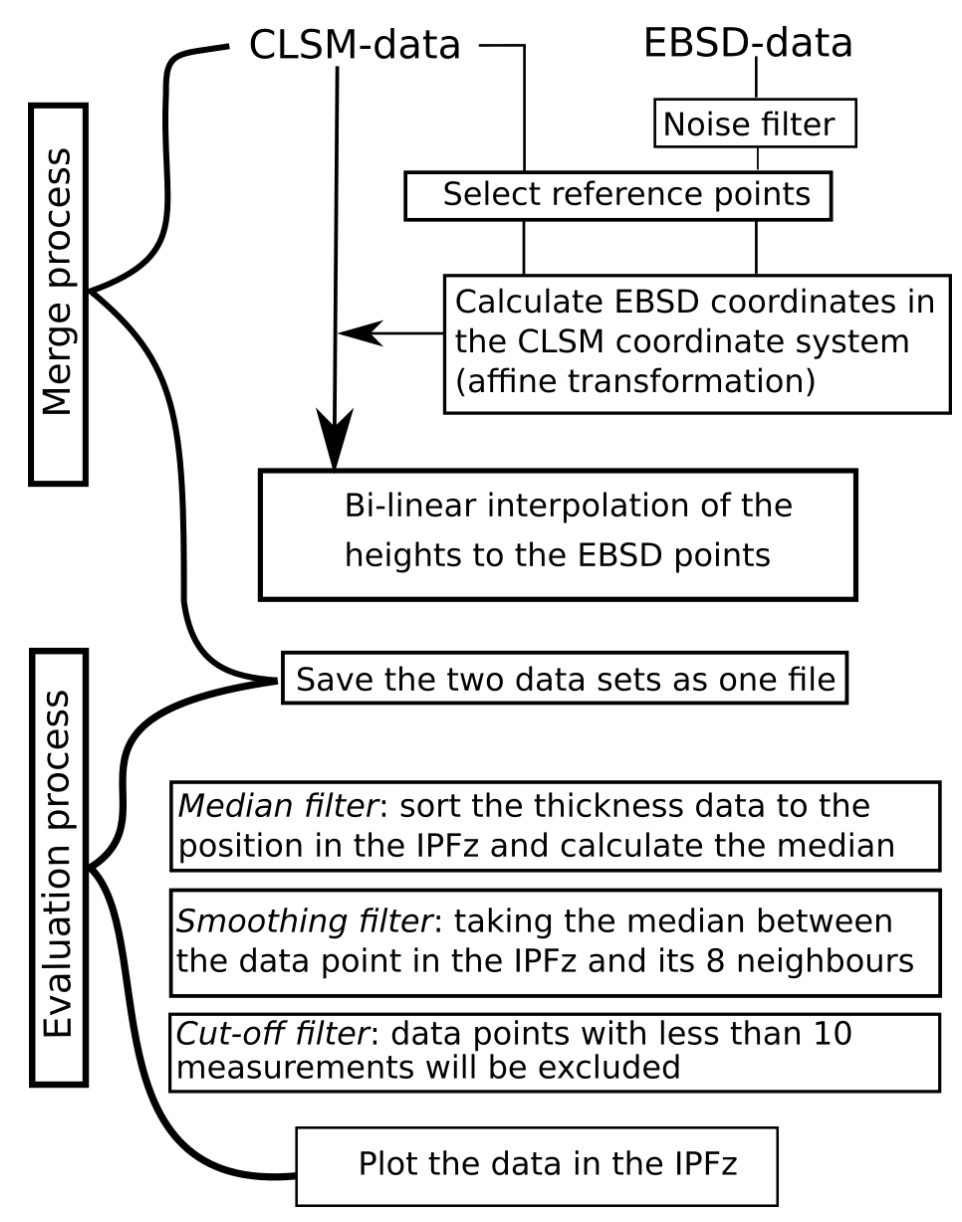

Figure 1: Flow chart of the python tool illustrating the merging and evaluation process. 


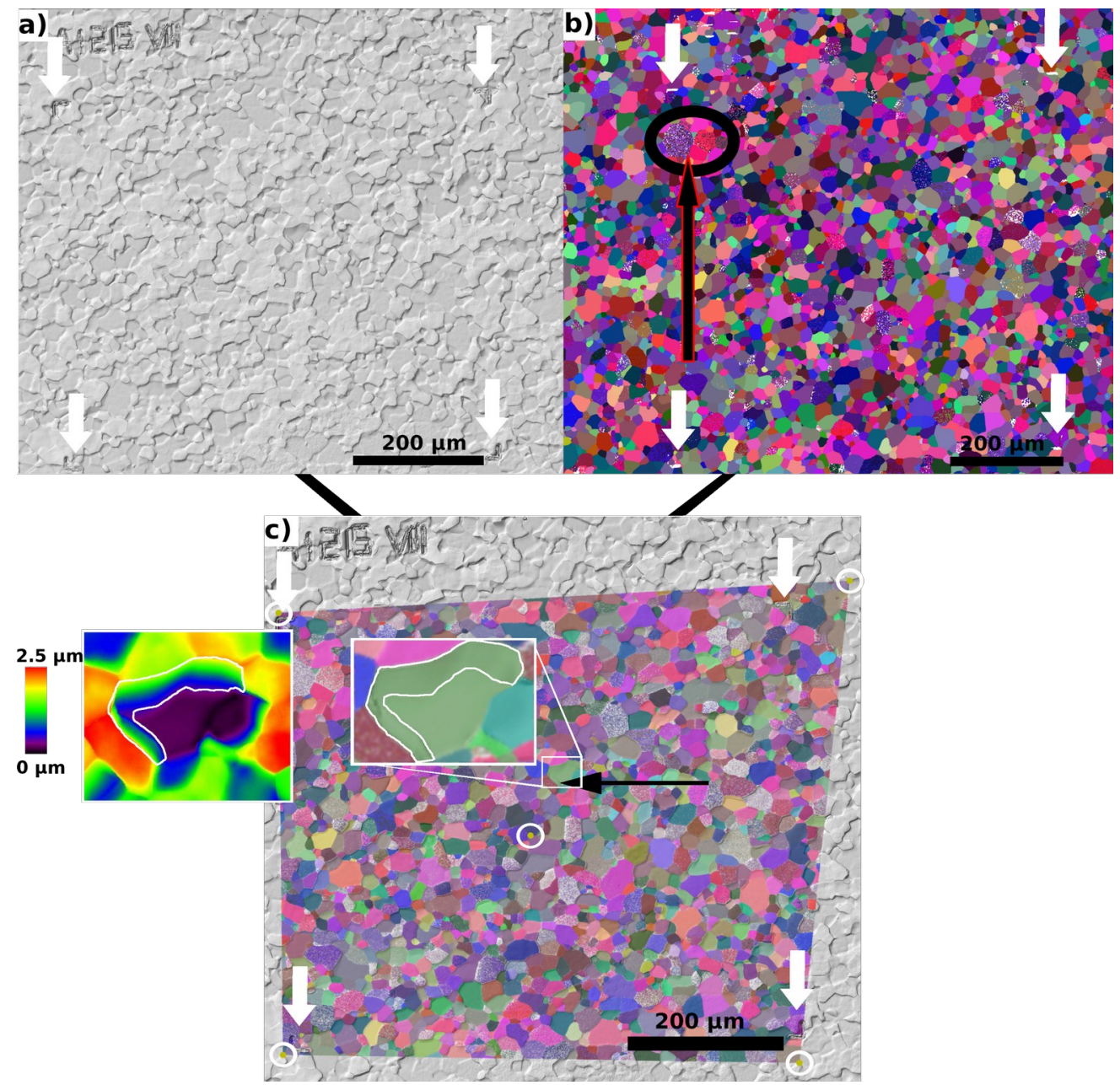

Figure 2: (a) CLSM height map in gray scale. (b) orientation map which shows the 3 Euler angles color-coded. (c) visualization of the merged data sets from $(a) /(b)$ illustrating the merging quality of the data from the two different microscopes. Four L markers on a tungsten sample are used for aligning the EBSD and CLSM data (marked by white arrows). In addition to these markers, the sample name is labeled with FIB. In (b), the black arrow with the ellipse shows a noisy EBSD area, which will be eliminated during the evaluation. In (c), the 4 yellow points at the corners as well as one yellow point in the middle (marked by white circle) are the selected positions for the merging procedure of the data from the two microscopes used in this example. The black arrow points to a pronounced grain boundary, which is influenced by the neighboring grains. The influence of the area is marked by a white line in the magnified overlay picture as well as in a colored height profile map (left). 


\section{Texture A}

a)

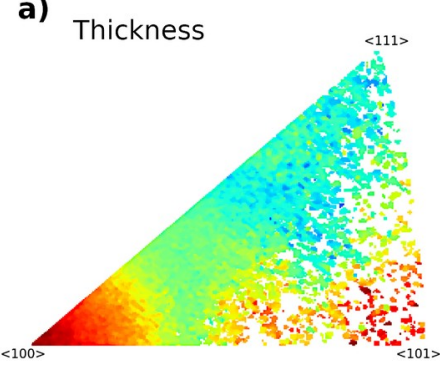

d)

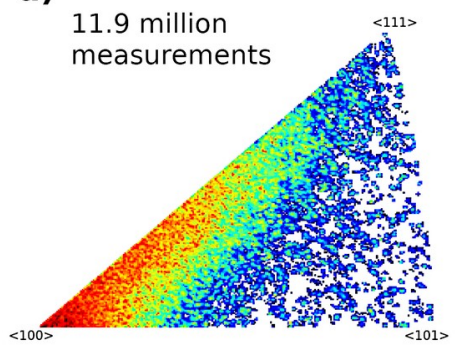

Texture B

b)

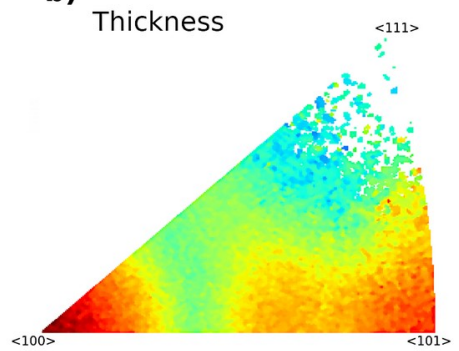

e)

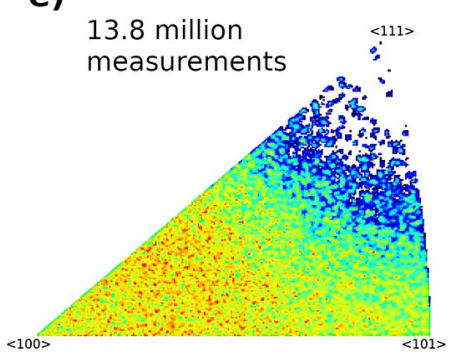

Texture A+B
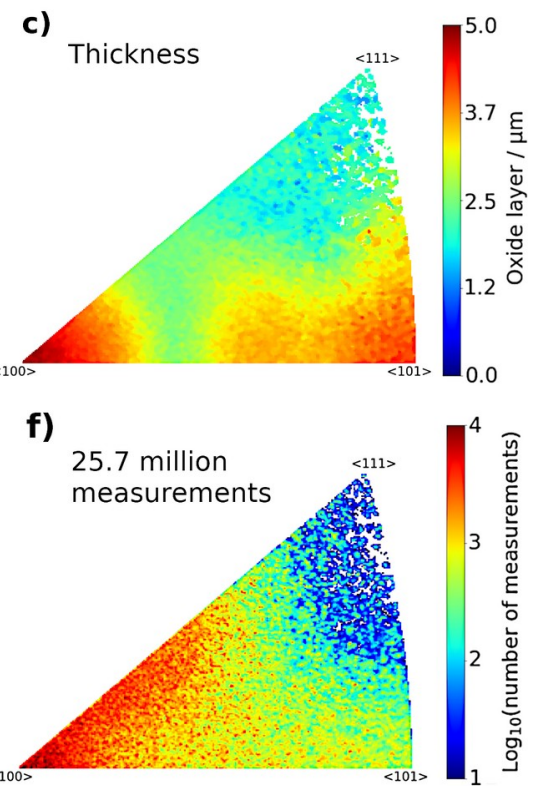

Figure 3: (a)-(c) thickness of the oxide layer versus the crystal orientation after applying all filters (see text). (d)-(f) number of measurements versus the crystal orientation. Each median of a pixel in (a)-(c) contains often thousands of thickness measurements. Samples with texture $A$ and B are oxidized at $870 \mathrm{~K}$ for 30 min. Median of the thickness measurements/number of measurements of: texture $A(a) /(d)$ and texture $B(d) /(e)$, all samples $(c) /(f)$.

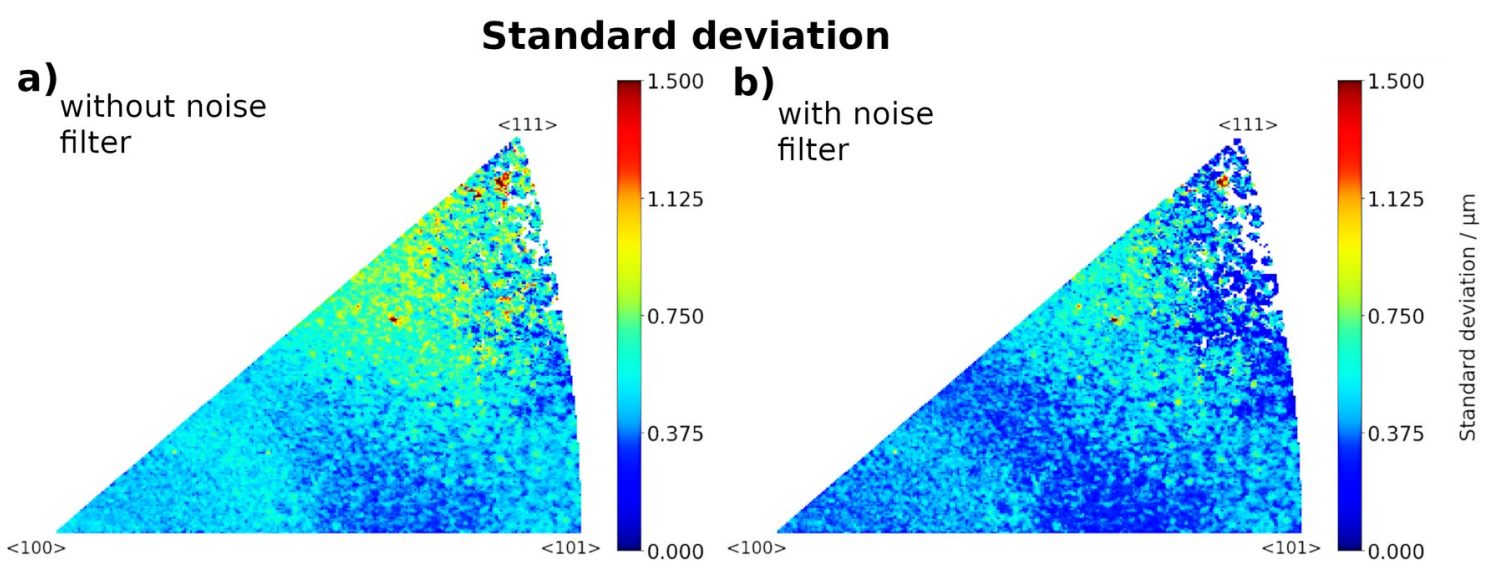

Figure 4: Standard deviation of the height measurement in fig. 3(c). Each pixel in fig. 3(c) is the median of often over 1000 measurements. (a) the standard derivation without a noise filter of the EBSD data. The average of standard deviation over all crystal orientation is $0.5 \mu \mathrm{m}$. (b) the standard derivation with a noise filter of the EBSD data. The average of standard deviation over all crystal orientation is $0.4 \mu \mathrm{m}$. 

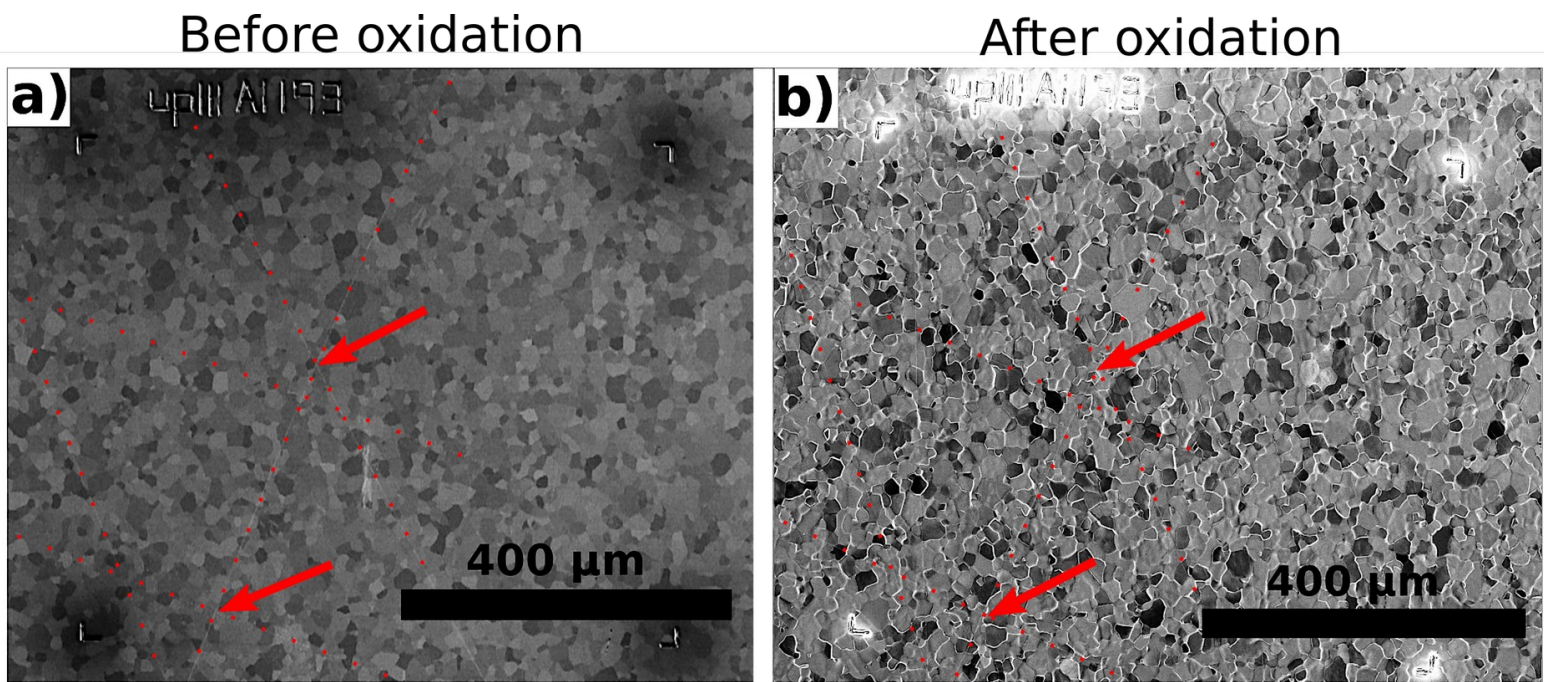

Figure 5: (a) SEM picture of a recrystallized tungsten sample before the oxidation experiment by detecting secondary electrons. (b) $\mathrm{Ga}^{+}$-ion picture by detecting secondary electrons after oxidation of 870 $K$ for 30 min of the same area as shown in (a). The different brightnesses in picture (b) indicates that the oxide layer on different tungsten grain is different. The red dotted point lines and arrows in both pictures mark scratches (hardly visible), which are visible before and after the oxidation. After oxidation, the scratches are not detectable in the CLSM height profile map, light microscope picture or intensity picture of the laser. 